\title{
3 Wie Wertschöpfung messen? - Anmerkungen zur Methodik und Datengrundlage
}

Die Grundannahme bei der Berechnung der nachfrageseitigen Wertschöpfung lautet, dass das Budget einer Hochschule über unterschiedliche Wege in die betreffende Region fließt (Maier et al. 2006): (1) durch die Beschäftigung, die die Hochschule als Arbeitgeber schafft; (2) durch unmittelbare Einkommenseffekte für die Angestellten der Hochschule sowie (3) durch Investitions- und Sachausgaben der Hochschule. Darüber hinaus entstehen noch die Einkommens- und Beschäftigungseffekte (4), die durch Konsumausgaben der Studierenden entstehen. Dabei geht jede Wertschöpfungsstudie von der Grundannahme aus, dass die Finanzmittel in der Region positive Einkommens-, Beschäftigungs- und Wachstumseffekte auslösen.

\section{Zur Methode der Schätzung regionaler Wertschöpfung}

Diese Ausgaben - nach verschiedenen Ausgabenarten, etwa den differenzierten Konsumausgaben der Studierenden - bilden mit den Beschäftigtenzahlen die direkten Wertschöpfungseffekte der Universität im Bereich Einkommen und Beschäftigung ab, müssen aber um räumliche, verzerrende Effekte - etwa Pendlerverflechtungen durch Hochschulbedienstete, die im Wiener Umland leben, oder Kaufkraftzu- und -abflüsse der Konsumausgaben - der Stadt zugeordnet werden (vgl. Abbildung 3.1). Die Grundlagen für diese Präzisierung der wertschöpfungsrelevanten Ausgaben werden in den Kapiteln 5 und 6 dargestellt - hier werden die Rohdaten der Hochschulen analysiert

Abbildung 3.1: Einflussfaktoren auf die wertschöpfungsrelevanten Ausgaben von Studierenden und Hochschulbediensteten

\begin{tabular}{|c|c|}
\hline $\begin{array}{c}\text { Ausgaben der Studierenden } \\
\text { (Studierenden- } \\
\text { sozialerhebung 2009/2011) }\end{array}$ & $\begin{array}{r}\text { Einkommen der HS-Bediensteten } \\
\text { (Konsumerhebung } \\
\text { der Statistik Austria) }\end{array}$ \\
\hline \begin{tabular}{|l|}
\hline \multicolumn{2}{|c|}{ - Keschlechterproportion } \\
- Tagespendler \\
- nicht kaufkraftrelevante Ausgaben \\
(etwa Miete)
\end{tabular} & $\begin{array}{l}\text { - 7 Einkommensgruppen } \\
\text { - Kaufkraftzu- und -abflüsse } \\
\text { - Tagespendler } \\
\text { - nicht kaufkraftrelevante Ausgaben } \\
\text { (Miete, Energie) }\end{array}$ \\
\hline $\begin{array}{c}\text { Kaufkraftrelevante Ausgaben } \\
\text { der Studierenden }\end{array}$ & $\begin{array}{l}\text { Kaufkraftrelevante Ausgaben } \\
\text { der HS-Bediensteten }\end{array}$ \\
\hline
\end{tabular}

Quelle: eigene Darstellung 2013. 
und regionalisiert (Stufe 1 und 2 in Abbildung 3.2) und werden anschließend in Kapitel 7 zusammengefasst. Aus diesen wertschöpfungsrelevanten Ausgaben lassen sich die direkten Einkommens- und Beschäftigungseffekte ableiten (Stufe 3), die die Grundlage für die weitere Schätzung bilden. In einem weiteren Schritt werden mittels eines Arbeitsplatzkoeffizienten die durch die Ausgaben (von Studierenden, Beschäftigten sowie den Sachausgaben der Hochschulen) entstehenden Effekte auf den Arbeitsmarkt geschätzt - es handelt sich hier um die indirekten Beschäftigungseffekte. Eine detaillierte Beschreibung der Methodik sowie die Ergebnisse sind in Kapitel 9 dargestellt. Abschließend werden auf der Grundlage der nationalen Input-Output-Tabelle jene Wertschöpfungseffekte geschätzt, die durch die gestiegene Beschäftigung in den jeweiligen Branchen in einer zweiten Wirkungsrunde entstanden sind (induzierte Einkommenseffekte, Stufe 5 in Abbildung 3.2).

\section{Abbildung 3.2: Schematische Darstellung für die Schätzung der Wertschöpfungseffekte}

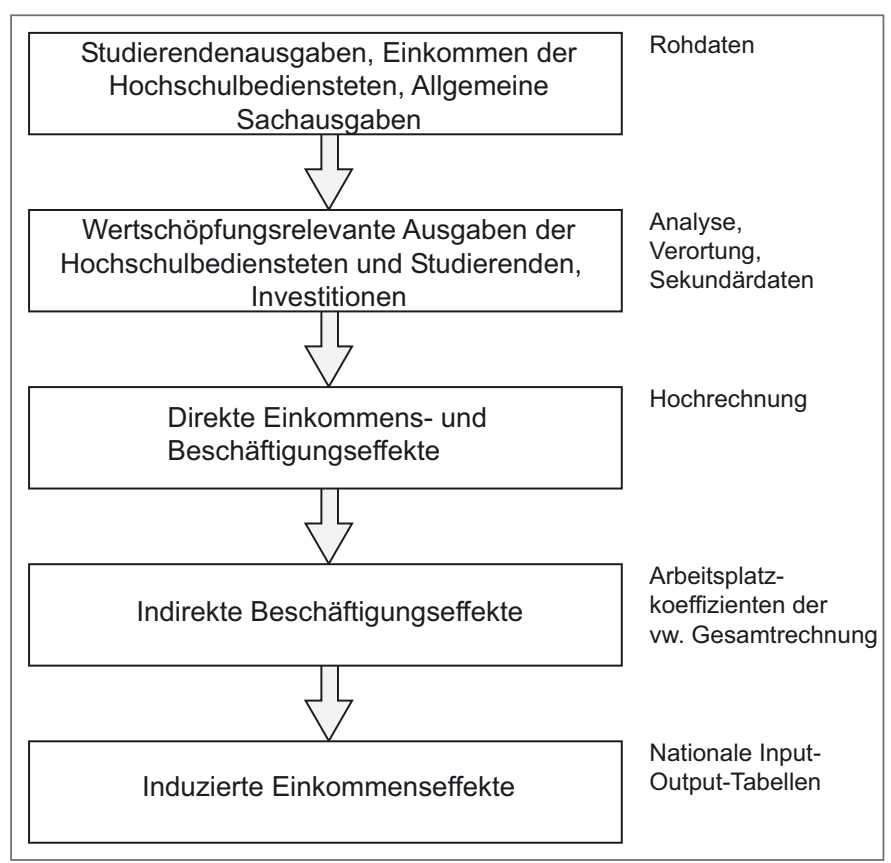

Quelle: eigene Darstellung 2013.

Diese Schätzwerte basieren überwiegend auf der Anwendung nationaler Multiplikatoren, da eine regionale Zuordnung problematisch ist. Dazu ein anschauliches Beispiel: Beliefert ein Wiener Bäcker die Universität Wien mit seinen Waren, so ergibt sich die direkte regionale Wertschöpfung durch den gestiegenen Umsatz des Bäckers, der damit mehr Arbeitnehmer beschäftigen kann (indirekter Arbeitsplatzeffekt). Um den induzier- 
ten Wertschöpfungseffekt für spätere Wirkungsrunden bestimmen zu können, ist zu klären, ob der Bäcker seine Vorprodukte aus Wien oder aus anderen Regionen bezieht. In Metastudien wird darauf hingewiesen, dass die geschätzten bzw. angenommenen regionalen Multiplikatoreffekte (etwa für regionale Importquoten) beträchtlich schwanken und einer gewissen Willkür unterliegen (Siegfried et al. 2006). Da verlässliche regionale Multiplikatoreffekte also nur durch umfangreiche Unternehmensbefragungen bestimmt werden können, greift diese Studie auf Koeffizienten zurück, die die nationale Konsumund Sparneigung sowie die nationale Importquote abbilden.

In dieser Studie wird die regionale Wertschöpfung zwar nach quantitativen, aber nicht ausschließlich nach monetarisierbaren Indikatoren bewertet. So wird der Bedeutung der Wiener Hochschulen für die Zuwanderung aus dem restlichen Österreich, aber auch aus dem Ausland nachgegangen. Wie zu zeigen sein wird, beeinflusst dies sowohl die demographische Struktur der Stadt als auch deren Migrationsregime. Der „demographische Impact“ kann durchaus beträchtlich sein und aufgrund der räumlichen Konzentration von Studierenden in einzelnen Stadtteilen spezifische Gentrifizierungsprozessen, gewissermaßen eine „Studentification“ auslösen (vgl. Hubbard 2008, Smith und Holt 2007).

Dieser holistische Zugang hat den Nachteil, dass am Ende mehrere Einzelergebnisse vorliegen, die nicht unmittelbar verknüpft oder aufsummiert werden können. Allerdings bietet sich damit ein über die regionalökonomische Schätzung hinausgehendes und umfassenderes Bild der regionalen Wertschöpfung und der Bedeutung der Wiener Hochschulen für die Stadt Wien.

\section{Datengrundlage der Studie}

Diese Wertschöpfungsstudie basiert vorrangig auf einer Sonderauswertung von internen Datenquellen der Wiener Hochschulen. Die im Rahmen der Studie entwickelte Datenbank umfasst Informationen über die Herkunft von Studierenden (In- und Ausland), die Beschäftigtenstruktur (Wohnort, Herkunft, Einkommensstufe, Qualifizierungsniveau, ...), wissenschaftliche Veranstaltungen an den jeweiligen Hochschulen sowie budgetäre Angaben, etwa über Drittmittel, Investitionen oder Sachausgaben. Von den 18 Universitäten, Fachhochschulen und Privatuniversitäten am Standort Wien haben elf an dieser Erhebung teilgenommen. Unvollständige Angaben sowie die Daten der restlichen Hochschulen wurden, wo es notwendig und inhaltlich sinnvoll war, geschätzt und ergänzt.

Da diese Studie auf den Angaben der einzelnen Hochschulen basiert, können sich gewisse Unschärfen dadurch ergeben, dass Studierende an mehreren Einrichtungen immatrikuliert sind und damit mehrfach gezählt werden. Weiters konnte nicht berücksichtigt werden, ob es sich bei den Studierenden um prüfungsaktive oder um sogenannte „Karteileichen“ handelt. Hier waren die Autoren auf die Angaben der Hochschulen sowie der amtlichen Statistik (uni:data) angewiesen. Aus diesem Grund ist es möglich, dass es zwischen den Statistiken zu Abweichungen aufgrund der Schätzungen kommen kann. So wurde beispielsweise die Herkunft der ausländischen Studierenden in Abstim- 
mung mit den uni:data-Angaben nach Weltregionen geschätzt. Auf nationalstaatlicher Ebene wurde hingegen von einer Schätzung aufgrund der zu hohen Ungenauigkeiten abgesehen.

Neben den hochschulinternen Daten bildete die amtliche Statistik eine wichtige Ergänzung und Referenz. So wurden beispielsweise die Angaben zur Staatsbürgerschaft oder der Heimatadresse der Studierenden auf die uni:data-Angaben hochgerechnet, womit eine Vergleichbarkeit zwischen den Hochschulen möglich ist. Weiters wurde für Informationen zur Bevölkerungs- und Wirtschaftsstruktur sowie zum Konsumverhalten auf Daten der Statistik Austria zurückgegriffen. Die induzierten Wertschöpfungseffekte wurden mittels der nationalen Input-Output-Tabelle der Statistik Austria berechnet.

Eine weitere wichtige, sekundäre Datenquelle stellte die Studierendensozialerhebung der Jahre 2009 und 2011 dar. Da im Rahmen dieser Studie keine eigene Erhebung zum Konsumverhalten der Studierenden gemacht wurde, erfolgte eine Schätzung der wertschöpfungsrelevanten Ausgaben auf Grundlage der Studierendensozialerhebung.

Die Abgrenzung der Stadtregion Wien erfolgte auf Basis der Erreichbarkeitsdaten, die der Verkehrsbund Ostregion dankenswerterweise zur Verfügung gestellt hat. Für die Berücksichtigung der Kaufkraftflüsse zwischen Wien und Niederösterreich konnte auf Erhebungen der Wirtschaftskammern von Wien und Niederösterreich zurückgegriffen werden. 